\title{
MOTIVOS DE PARTICIPACIÓN Y SATISFACCIÓN EN LA ACTIVIDAD FÍSICA, EL EJERCICIO FÍSICO Y EL DEPORTE
}

\author{
Elmer Garita Azofeifa \\ Maestría Ciencias del Movimiento Humano. \\ Escuela de Educación Física y Deportes, Universidad de Costa Rica. \\ E-mail: garitaazofeifa13@gmail.com
}

\section{RESUMEN}

\begin{abstract}
La motivación en la actividad física es una característica psicológica multidimensional, que se ve influenciada tanto por los aspectos internos de la persona (preferencias, deseos, temores, entre otras), como por las vivencias externas de su entorno (aceptación social, amistades, habilidades, entre otras). En una época en la que en el nivel mundial, la actividad física de personas de todas las edades, está en aumento constante, es fundamental para profesionales como educadores físicos, entrenadores deportivos o instructores de ejercicio, tener presentes los principales motivos de participación de los sujetos que tienen a su cargo, con el fin de velar por elaborar planes de trabajo ideales para que se mantengan realizándolo, orientándolos a disfrutar y estar satisfechos con su intervención y así alargar su ciclo de vida activa, evitando el abandono, aspecto tan relacionado con el sedentarismo y el riesgo de padecer enfermedades crónicas y degenerativas. Así, los niños prefieren la diversión y hacer amigos; los adolescentes la competición y las amistades; los universitarios la aventura y la diversión; los adultos obtener actividad física regular y los adultos mayores los beneficios relacionados con la salud. Las mujeres se motivan por la apariencia y aspectos sociales, mientras los hombres por la competición y el estatus. Los sujetos que hacen deporte se motivan por la competición y los que hacen ejercicio por la imagen corporal. A mayor nivel de actividad, se valora más la competición. Finalmente, la diversión, la competición, el aprender habilidades y la condición física son los motivos más importantes para participantes de actividad física norteamericanos, europeos y asiáticos. El presente trabajo se realizó con el propósito de dar a conocer a los profesionales de las ciencias del movimiento humano, las diferentes variables que determinan los motivos de participación en los distintos tipos de actividad física en sujetos con diferente edad, género, cultura y nivel de actividad.
\end{abstract}

PALABRAS CLAVES: actividad física, cultura, deporte, ejercicio, motivación, motivos para participación, satisfacción.

\author{
REASONS FOR PARTICIPATION AND SATISFACTION IN \\ PHYSICAL ACTIVITY, PHYSICAL EXERCISES, AND SPORTS
}

\section{ABSTRACT}

Motivation in physical activity constitutes a multidimensional psychological characteristic that is influenced by the person's internal aspects (preferences, desires, fears, etc.) and his/her experiences in the external environment (social acceptance, friends, skills, etc.). In a period in which physical activity is globally increasing among people of all ages, it is important for physical educators, sports trainers, or physical instructors to know the main reasons for their trainees to exercise with the purpose of preparing ideal workout plans that would help them continue exercising. These plans should encourage subjects to enjoy and be satisfied with their participation, therefore, extending their active life cycle and avoiding quitting, which are closely related to a sedentary lifestyle and the risk of having chronic and degenerative diseases. Consequently, children prefer to exercise to have fun and make friends, adolescents to compete and make friends, college students for adventure and fun, adults to have regular physical activity, and senior citizens to obtain health benefits. Women are motivated by their appearance and social reasons, while men do it for competition and status. Subjects who practice sports are motivated by competition, while those who exercise do it for body image. The more physical activity is practiced the more value is given to competition. Finally, having fun, competing, learning skills, and being in good physical condition are the most relevant reasons for American, European, and Asian subjects to participate in physical activity. This research was conducted with the purpose of letting professionals of human movement sciences know the variables that determine the reasons for subjects of distinctive ages, gender, culture, and level of activity to participate in the different types of physical activities.

KEY WORDS: physical activity, culture, sports, exercise, motivation, reasons for participation, satisfaction. 
El presente documento, tiene como propósito presentar a los profesionales en las ciencias del movimiento humano y de la salud, la descripción de los motivos que tienen las personas para participar en la actividad física, el ejercicio físico y el deporte, así como la satisfacción al llevarlas a cabo, tomando en cuenta los resultados encontrados por una extensa revisión de literatura en la que se incluyen como variables la edad, el sexo, la cultura y nivel de actividad, entre otras; esto, con el fin de que puedan conocer mejor las tendencias motivacionales de las personas a su cargo y así se promuevan planes de ejercicio y entrenamiento acordes con las necesidades de los mismos, ampliando la satisfacción hacia la práctica de la actividad física y evitando la deserción de los programas de ejercicio y deporte.

\section{Actividad física: diferencias entre ejercicio y deporte}

La actividad física ha empezado a incrementar en los últimos años ya que se la han atribuido efectos en la función fisiológica y en el bienestar mental y psicosocial. Existe evidencia de que al incrementar la actividad física se mejora la condición física y se producen cambios fisiológicos y psicológicos importantes (Asci, Kin y Kosar, 1998).

Como actividad física, se conoce todo aquel movimiento que realiza el ser humano que implica el desplazamiento de los diversos componentes corporales y el gasto energético que pueden ser realizados durante el diario vivir, como ocupación, distracción, ejercicio y deporte. El ejercicio, por su parte, es un tipo de actividad física, planeada y estructurada, en la que el hombre participa con el objetivo establecido de mejorar alguna de sus cualidades físicas como la fuerza, la potencia, la velocidad, la resistencia aeróbica, entre otras; $\boldsymbol{e l}$ deporte, es la actividad física organizada en ligas con competición individual o grupal (Nigg, 2003). Teniendo en claro esta diferencia, algunos estudios han usado el deporte como variable (Frederick y Ryan, 1992; Weinberg, Tenenbaum, McKenzie, Jackson, Ashel, Grove y Fogarty, 2000; Isogai, Brewer, Cornelius, Etnier y Tokunaga, 2003; Rosich, 2005), mientras otros lo han hecho con el ejercicio (Smith y Storandt, 1997; Asci y cols, 1998; Pavón, Moreno, Gutiérrez y Sicilia, 2003).

\section{Motivación}

Tanto en la actividad física como en cualquier ámbito de la sociedad, el éxito depende de muchos factores, ya sean de tipo ambiental, biológicos, de personalidad, psicológicos, entre otros. Uno de los aspectos más importantes para el buen desempeño en determinada actividad es la motivación, ya que funciona como factor regulador de la energía y la emoción empleada para cumplir un objetivo (Valdés, 1996) y es uno de los tópicos de investigación más comunes en psicología general o deportiva (Biddle, 1999); de hecho, Pelletier, Fortier, Vallerand, Tuson, Brière y Blias (1995), indican que la motivación es uno de los problemas deportivos más interesantes, tanto en la respuesta del rendimiento de ambientes sociales como la competición y el comportamiento de los entrenadores, como la influencia en el desarrollo de variables de comportamiento como la persistencia, aprendizaje y rendimiento.

Antes de ahondar en el tema de la motivación, es importante establecer su definición, la cual según Morilla (1994), es la intensidad y dirección del comportamiento; la dirección se refiere a si el individuo busca, se aproxima o siente atraído por ciertas situaciones y la intensidad se refiere a la cantidad de empeño que la persona emplea en una situación determinada (Weinberg y Gould, 1996). Además, se puede agregar que la persistencia, en el sentido de determinar cuánto dura el sujeto en la actividad, puede ser un indicador importante de motivación; entonces, el proceso motivacional es el que aporta energía, dirección y persistencia al comportamiento y está determinado por múltiples necesidades: biológicas, cognitivas, afectivas y sociales (Valdés, 1996).

El concepto de motivación es un término multidimensional, integrado por la motivación intrínseca, extrínseca y la amotivación. La motivación intrínseca se refiere a comprometerse en una actividad 
puramente por el placer y la satisfacción que provoca hacerla; cuando una persona está motivada intrínsecamente se comporta voluntariamente en ausencia de recompensas materiales u obligaciones o presiones externas (Deci y Ryan, 1985). En este tipo de motivación, se han identificado tres diferentes categorías: a.) Motivación intrínseca hacia el conocimiento, que está relacionada con la exploración, curiosidad, metas de aprendizaje, motivación de aprender por la necesidad de conocer y entender. Es desempeñar una actividad por el placer y la satisfacción que se experimenta mientras aprende, explora o trata de entender algo nuevo; b.) Motivación intrínseca hacia el cumplimiento, también llamado motivación de dominio, motivación de eficacia y orientación a la tarea y definida como comprometerse en una actividad por el placer y la satisfacción experimentada cuando se intenta crear algo o cumplir con alguna técnica de entrenamiento difícil y c.) Motivación intrínseca para experimentar estimulación, es cuando alguien se compromete en una actividad con el fin de experimentar sensaciones estimulantes producto de su participación, por ejemplo, un atleta que participa en el deporte para vivir experiencias emocionantes (Pelletier y cols., 1995). Una investigación exhaustiva se ha realizado en el tema de la motivación intrínseca, en los estudios de Glenn, Horn, Campbell y Burton (2003) y Huang, Chou, Lu, Chen, Yeh, Chang, Lin, Lin, Wang y Wu (2003).

Por el contrario, existe otro tipo de motivación en la cual el sujeto basa su energía para realizar la actividad en medios externos, es decir, su principal motivación no se encuentra dentro de sí, sino que proviene del exterior, por la recompensa material o social, entre otros; este tipo de motivación es llamada Motivación extrínseca, que no es un comportamiento determinado por si mismo, solamente puede ser propuesto por asuntos externos como las recompensas y se subdivide en: a.) Regulación externa, que se refiere al comportamiento que es controlado por medios externos, como recompensas materiales o la imposición de otros (Deci y Ryan, 1985); b.) Introspección, indica que quien participa en el deporte es porque siente presión de estar en buena forma por razones estéticas, porque se siente avergonzado o apenado cuando no está en su mejor forma y c.) Identificación, que es cuando el sujeto valora o juzga un comportamiento como importante y por eso lo elige. Siente que su participación contribuye a una parte de su crecimiento y desarrollo como persona (Pelletier y cols., 1995).

Por su parte, Biddle (1999) expresa que son cuatro los tipos de motivación extrínseca, los tres anteriormente mencionados además de regulación integrada, que es la más autodeterminada forma de regulación de comportamiento; el comportamiento es integrado dentro de su propia personalidad o identidad.

Finalmente, el análisis de la teoría motivacional sugiere que también existe un tipo de motivación, en la cual, el sujeto no es motivado ni interna ni externamente; en otras palabras, no tiene definida la motivación por la que practica el deporte; este tipo de indecisión motivacional es llamada Amotivación, que es cuando el individuo no percibe eventualidad entre sus acciones y las respuestas a sus acciones. Los sujetos amotivados, experimentan sentimientos de incompetencia y falta de control (Deci y Ryan, 1985). Además la amotivación ha sido hallada como el estado que existe cuando el individuo tiene poca o no tiene motivación para intentar un comportamiento (Biddle, 1999).

Así como son muchos los aspectos que provocan la deserción de deportistas (otras prioridades, diferencias con el entrenador, falta de tiempo, entre otros), el establecimiento de objetivos o metas es un factor que influye en la preferencia del uso de determinado tipo de motivación, esto quiere decir que algunos atletas eligen objetivos realmente retadores, difíciles, que demandan gran energía y tiempo para obtenerlos, enfrentando sus deseos y temores y algunos obstáculos que se presentan externamente; por otro lado, existen los deportistas que seleccionan sus metas en las cuales el esfuerzo aplicado para llevarlas a su fin no sea mucho, es decir, metas fáciles de alcanzar, donde no entreguen ni dediquen su mayor cantidad de energía e interés, no dan lo mejor de sí. El establecimiento de metas se ve influenciado por diferentes variables como el sexo, la edad y la cultura de los sujetos, en otras palabras, dependen de cada sujeto y de la situación específica que viva. 


\section{Teorías sobre motivación}

Según Weinberg y colaboradores (2000), los estudios en motivación deportiva han sido basados en la teoría y mencionan que los tres modelos teóricos con mayor utilidad han sido la teoría de la competencia motivacional (Harter, 1981), la teoría de la autodeterminación (Deci y Ryan, 1985) y la teoría de orientación de metas (Maehr y Nicholls, 1980; Nicholls, 1984).

La teoría de la competencia motivacional (TCM), debate que la base para la motivación intrínseca es el deseo de un individuo de demostrar competencia a través del dominio de experiencias, y el éxito en este aspecto le permite continuar motivado y participando (Harter, 1981).

La teoría de autodeterminación (TAD) similar a la TCM, ve la obtención de logros y el esfuerzo a través de la competencia, como la motivación, interés y disfrute principal para la participación. Sin embargo, se ha reconocido que los motivos tanto intrínsecos como extrínsecos, están operando potencialmente cuando el individuo está tomando una decisión sobre participar o continuar su participación en la actividad física o el deporte. Los individuos motivados intrínsecamente están motivados principalmente por participar de una actividad que les de competición y disfrute, mientras que los motivados extrínsecamente participan para obtener recompensas o premios, aspectos externos a su comportamiento (Deci y Ryan, 1985; Weinberg y cols., 2000).

La teoría de orientación de metas (TOM) sugiere que existen dos perspectivas de metas independientes en el contexto del logro: la orientación de metas a la tarea y la orientación de metas al ego. El sujeto orientado a la tarea, emplea mayor esfuerzo para obtener los logros personales porque sabe que entre más se esfuerce puede lograr mayor destreza en la tarea; juzga su nivel de habilidad en referencia a sí mismo, de acuerdo a cuanto ha aprendido o mejorado progresivamente; se percibe habilidoso cuando está teniendo éxito en la tarea porque se ve motivado principalmente por ser competente y por el dominio de destrezas (Cervelló y Santos-Rosa, 2001; Seifriz, Duda y Chi, 1992; Simons, Dewitte y Lens, 2003). Por su parte en la orientación al ego, el individuo pone sus metas para mostrar un rendimiento superior a los demás, por demostrar habilidad a través de la comparación social con otros individuos. Para percibir alta habilidad en este caso, el sujeto debería rendir más que otros en determinada tarea. Las situaciones caracterizadas por la competición interpersonal, la evaluación pública y el feedback obligatorio generan en su mayoría orientaciones al ego (Vlachopoulos, Biddle, Fox, 1996; Simons y cols., 2003; Cervelló y Santos-Rosa, 2001).

Duda y Hayashi (1998), indicaron que los deportistas jóvenes están principalmente orientados al ego y poco orientados a la tarea, es decir, los sujetos pueden ver sus concepciones de habilidad de diferente manera cuando han adoptado una perspectiva de logro sobre otra, podría estar alto o bajo en una orientación o en ambas.

Las orientación de metas han sido relacionadas con diferentes variables, como la satisfacción, preferencia por tareas de diferente dificultad, percepción de habilidad, disfrute, clima motivacional percibido y el grupo de pares, entre otros grupos de variables (Nicholls, 1989; Butt y Cox, 1992; Seifriz y cols., 1992; Carron, 1996; Valdés, 1996; Vlachopoulos y cols., 1996; White, 1998; Babbkes y Weiss, 1999; Weinberg y cols., 2000; Cervelló y Santos-Rosa, 2001; Chi, Lin, Hung, y Liu, 2002; Chi, Tung, Liu, y Feng, 2002; Weiss y Smith, 2002; Brunel, 2003; Glenn y cols., 2003; Isogai y cols., 2003; Kim, Williams y Gill, 2003; McCarthy, White y Amorose, 2003; Rudisill, Wall, Parish, St Onge y Goodaway, 2003; Simons y cols., 2003; Smith, Ullrich-French, Walter y Hurley, 2003; Etnier, Sidman y Hancock, 2004). 


\section{Motivos de participación en actividad física}

Una de las áreas más investigadas dentro de la psicología del deporte y el ejercicio físico, es el referente a los principales motivos y razones por las cuales los niños o los jóvenes se inician en el deporte y permanecen en él o por el contrario, abandonan la práctica deportiva.

El origen de esta línea de investigación se remonta a 1938 cuando aparece la lista de Murray, compuesta por 12 motivos de necesidades biológicas y 28 de necesidades psicológicas para practicar deporte (Martínez, Andrade y Salguero, 2005). Años más tarde, un investigador americano (Skubic, 1956) realizó un trabajo con beisbolistas, en el que encontró que participar con otros muchachos, divertirse y superarse, fueron las razones citadas con mayor frecuencia para practicar el deporte; con lo que se considera éste, como el estudio pionero de motivos de participación deportiva.

Antes de que Gill, Gross y Huddleston (1983), publicaron el "Participation Motivation Inventory", los instrumentos para medir los motivos de participación eran inespecíficos; este hecho, contribuyó a que el auge investigador del tema en el contexto deportivo aparezca alrededor de los años 80 (Martínez y cols, 2005).

En una época en la que a nivel mundial, la participación de niños y jóvenes en diferentes disciplinas deportivas está en aumento constante, Cruz (1997), indica que los niños y los jóvenes han dedicado y gozado en todas las épocas una parte importante de su tiempo al juego y a una gran variedad de deporte, de hecho, muchos niños y niñas prefieren la práctica deportiva a cualquier otra actividad en su tiempo libre. Entonces se crea la necesidad de investigar a qué se debe tan importante fenómeno, conocer cuáles son las razones primordiales por las que los niños ingresan a los programas deportivos o recreativos y hasta que punto lo hacen por gusto propio o son llevados por deseo de sus padres.

Gill y cols. (1983) expresaron que en los años recientes, los investigadores y en especial los psicólogos, han mostrado un creciente interés en el deporte de los niños, sin embargo, el conocimiento no ha llegado muy lejos por lo que se amerita más investigación; similarmente, Gould (1982), recomienda que existen dos aspectos psicológicos requeridos para estudios futuros: determinar porqué los jóvenes atletas participan en deportes y entender porqué los jóvenes atletas dejan de participar.

Los motivos de participación en la actividad física son de diversa índole; el Cuestionario de Actitudes, Motivaciones e Intereses hacia las Actividades Físico-Deportivas (Pavón y cols, 2003) los delimitan en 6 motivos: motivos de competición; capacidades personales; aventura; hedonismo y relación social; forma física e imagen personal y salud médica. Por su parte el Cuestionario sobre Motivos de Participación Deportiva (Durand, 1988), mide los motivos en ocho factores: autorrealización o motivación de status; diversión; liberación de energía; búsqueda de esparcimiento; desarrollo de habilidades; factores sociales; práctica en equipo y forma física. Por su parte, Recours, Souville, y Griffet (2004), proveen una escala para medición de cuatro dimensiones de motivación deportiva: exhibicionismo, competición, sociabilidad y emoción y jugando al límite.

Los motivos que aparecen con mayor consistencia en la literatura, incluyen la diversión, el desarrollo de habilidades, condición física, incorporación, reto, éxito, estatus y el aprendizaje (Weinberg y cols, 2000; Gill y cols., 1983).

Se aprecia entonces lo complejo que resulta el abordaje de este tema, en el que influyen factores como las diferencias socioculturales, el deporte practicado, el género, la edad de las poblaciones estudiadas y la satisfacción al practicarlas. 


\section{Satisfacción}

"La diversidad de actividades físicas y deportivas es tal que en ella puede encontrar satisfacción toda la gama de las necesidades humanas, y en cierto sentido, estudiar las motivaciones de quienes practican deporte significa estudiar las motivaciones del hombre en general" (Durand, 1988, p.88). Se han reportado múltiples factores personales (edad, género, mentalidad, personalidad, nivel educativo, ocupación, propiedades sociales y culturales), y factores ambientales (trabajo, medio ambiente, condiciones de trabajo, familia, entre otros) que son importantes para obtener diferencias en satisfacción en humanos (Yilmaz y Akandere, 2003; Trail, Anderson, y Flink, 2002); tener altos niveles de satisfacción durante la vida, es fundamental para ser una persona saludable, exitosa, feliz y productiva. Existen tres variaciones en la satisfacción para vivir: necesidades, valores y expectativas. Las necesidades se pueden definir como las cosas que son muy importantes para el organismo para vivir saludable; los hay primarios (como comida, agua, sexo y dormir) y secundarios (sociales como el amor); mientras que los valores se definen como las esperanzas de sus propósitos, intereses y requerimientos y finalmente, las expectativas que están relacionadas con lo que la persona espera obtener o llegar a ser (Yilmaz y Akandere, 2003).

Rosich (2005), expresa que los niveles de satisfacción personal provocados por el ejercicio son de diversa índole; tales como físicos, relacionados con el bienestar fisiológico y psíquico/psicológico. En diversos estudios relacionados con el tema de satisfacción se ha demostrado que los sujetos orientados a la tarea están más satisfechos e interesados en practicar y disfrutar el deporte que los orientados al ego (Cervelló y Santos-Rosa, 2001); en mujeres adolescentes orientadas a la tarea, la satisfacción fue significativa cuando trabajaron experiencias relacionadas con el mejoramiento de la técnica o de las destrezas físicas, así como las que promovieron la aprobación social de otros; por su parte en este mismo grupo de adolescentes basketbolistas, la satisfacción se derivó del éxito normal, de ser mejores que los demás (Treasure y Roberts, 1998). El factor de satisfacción física-psíquica-social entre los deportistas que pertenecen a alguna federación o asociación deportiva y los no federados, mostró que en ambos grupos se encontraron altos niveles de satisfacción en hombres y mujeres, siendo mayor el de las mujeres federadas, aunque se constata una mayor satisfacción con la competición de los que están federados, sin obtener diferencias significativas entre hombres y mujeres (Rosich, 2005); cabe agregar que estas últimas presentan mayor insatisfacción corporal (Trew, Scully, Kremer y Olge, 1999).

\section{Diferencias de edades}

Taylor, Blair, Cummings, Wun y Malina (1999) investigaron la influencia de la experiencia relacionada con la participación en actividades físicas durante la niñez, adolescencia y el nivel de actividad física de adultos. Campbell, MacAuley, McCrum y Evans (2001), estudiaron las diferencias de edad en los factores de motivación para el ejercicio y encontraron que tanto para el grupo de edades entre los 16 y los 44 años como el de edades entre 45 y los 74 años, el 90\% de los sujetos expresó que el factor más importante es "sentirse físicamente en buena forma".

La motivación de un niño puede ser condicionada por sus padres y un tiempo después, por su entrenador, por lo que éste se convierte en una figura de referencia también para los padres. Una relación positiva de los niños con el entrenador, quien actúa como guía y facilitador de su proceso de enseñanza, en la cual los niños son respetados y estimulados a desarrollar sus personalidades, también es un factor motivante que satisface las necesidades de los niños de tener una referencia de adulto (Bergerone, Cei y Ceridono, 1985).

La investigación descriptiva ha contribuido a entender porqué los niños participan en deporte. Esta línea de investigación indica que los niños tienen múltiples motivos para continuar participando, entre 
los cuales se encuentran ser competente, la amistad, el desarrollo de habilidades y la competición (Kim, Chang y Gu, 2003; Wang y Weise-Bjornstal, 1996).

Los niños podrían participar en el deporte por una combinación entre motivos intrínsecos y extrínsecos, aunque muchos sugieren que en su mayoría son motivaciones intrínsecas, ya que es algo retador y disfrutable, así como también participan por motivos extrínsecos como ganar la aprobación externa (Weigand y Broadhurst, 1998) y gustar al entrenador, motivo que resulta más importante cuando más pequeños son (Williams y Cox, 2003). Para Hagger, Cale y Almond (1997), los niños tienen actitudes más positivas hacia la actividad física principalmente durante los meses de verano, sin establecer diferencias de género.

González, Tabernero y Márquez, (2000), encontraron que tanto niños futbolistas como tenistas concedieron mayor importancia a los siguientes motivos para practicar deporte: mejorar el nivel, estar bien físicamente, divertirme, hacer ejercicio, mantenerse en forma, aprender nuevos movimientos o habilidades y hacer nuevos amigos; resultados que están de acuerdo con lo encontrado por Gould, Feltz y Weiss (1985), lo que demuestra que independientemente del deporte practicado, los motivos para practicar deporte son similares, dejando claro que los jóvenes deportistas se acercan más al deporte por motivos intrínsecos que extrínsecos. Contradiciendo lo anterior, Weinberg y cols. (2000), encontraron que las razones más mencionadas fueron extrínsecas y relacionadas con la competición, así como han mostrado que la afiliación social, el estatus y el reconocimiento son importantes razones extrínsecas por las que los niños y adolescentes participan en deportes.

Estudios epidemiológicos en Estados Unidos y en Canadá indican que con el aumento en la edad, el comportamiento hacia el ejercicio tiende a disminuir, especialmente en los años de la adolescencia (Nigg, 2003). Al llegar la adolescencia disminuye la actividad física en ambos sexos pero principalmente en las mujeres, por el asunto de la pérdida de feminidad y la discriminación asociada a su participación en el deporte (Cale, 1996; Cookburn, 2000).

En cuanto a los jóvenes, entre los principales motivos de participación se menciona la diversión (Campbell y cols., 2001; Weinberg y cols., 2000), aprender nuevas habilidades (Weinberg y cols., 2000), mejorar su forma física (Rosich, 2005), competir (Hellandsig, 1998; Nigg, 2003) y cooperar con otros, aprender a tomar riesgos y a auto-manejarse, la posibilidad del éxito y el fracaso (Nigg, 2003) y obtener estatus, prestigio y reconocimiento (Alderman y Wood, 1976; Williams y Cox, 2003).

Los universitarios pasan por un periodo crítico en cuanto a la participación deportiva, ya que es aquí donde la gran mayoría de estudiantes de ese nivel abandonan el hábito de hacer ejercicio, pues deben dedicar muchas más horas al estudio y reducir las de ocio (Blasco, Capdevilla, Pintanel, Valiente y Cruz, 1996). Evaluando la percepción de los estudiantes universitarios según su nivel de actividad, expresan diversos motivos de participación deportiva, entre ellos: la competición, la capacidad personal, las relaciones sociales, aventura, forma física e imagen personal (Pavón y cols., 2003). Si se desea un incremento en el nivel de motivación de estudiantes universitarios para la práctica, se debe hacer hincapié en las habilidades básicas, en la diversión y en las relaciones grupales, no tanto en la popularidad, la recompensa externa y la competición (Tabernero, 1998).

Paralelamente a lo que sucede con los universitarios y debido a que se da en un periodo cronológico similar, parece que el adulto joven pasa por una etapa crítica para estimar la continuación en la participación deportiva, ya que la adherencia al ejercicio disminuye con la edad y un tercio de la población de adultos reporta no tener una rutina de ejercicio regular (Smith y Storant, 1997).

Está claro que las enfermedades son una barrera para que el adulto mayor se ejercite, y la inactividad física produce deterioro en la condición física, incluso, estas personas se hacen dependientes de otras en actividades de su diario vivir. Un programa de actividades multidimensional que incluya resistencia, 
fuerza, balance y entrenamiento de la flexibilidad es considerado generalmente óptimo para adultos mayores (Cress, Buchner, Phohaska, Rimmer, Brown, Macera, DiPietro y Chodzko-Zajko, 2005). En la mayoría de los estudios con adultos mayores los principales motivos para participar suelen ser directamente los relacionados con la salud (Rosich, 2005; Weinberg y cols., 2000; Smith y Storant 1997), no así en un estudio, donde el $87.8 \%$ de los sujetos, expresaron que es tarde para prevenir; además, muy pocos adultos en comparación con los jóvenes, creen que el ejercicio puede ayudarles a divertirse y por el contrario, sentirse mentalmente alerta o salir de casa lo consideran su prioridad (Campbell y cols., 2001), así como también la condición física y la relajación (Weinberg y cols., 2000). Según Smith y Storant (1997), los adultos inmersos en la competición deportiva presentan una mayor variedad de razones para participar como la socialización y la competitividad mientras que quienes hacen ejercicio no competitivo y quienes son sedentarios le dieron prioridad a los beneficios del ejercicio sobre la salud. Además, ambos grupos activos expresaron que el ejercicio es un medio para reducir el estrés y mejorar el estado de ánimo, por lo que los beneficios del ejercicio sobre la salud mental están claros solamente para los grupos de adultos mayores físicamente activos.

\section{Diferencias de sexo}

Gould y cols (1985), encontraron que las diferencias entre sexos se deben básicamente a que los niños participan por estatus social y aunque las posibilidades para las niñas aumentan, los niños tienen más opción de obtener mejores oportunidades deportivas principalmente en el nivel profesional. Tercedor y Delgado (1998), encontraron que en relación al género, las niñas de edad escolar, en comparación con los niños, siempre fueron inferiores en actividad física practicada y en el nivel de actividad física. Por su parte, en un estudio realizado en niñas chinas, se mostró que éstas, dieron tanta importancia a la orientación de equipo como los niños le dieron mayor influencia a la condición física, al gasto energético y a la amistad (Wang y Weise-Bjornstal, 1996; Kim y cols., 2003a).

Las mujeres, suelen tener metas de control de peso y mejorar su apariencia (Rosich, 2005) y han demostrado estar orientadas a lo social, lo que sugiere que dan mayor importancia a la relación de pares y un fuerte énfasis social (Isogai y cols., 2003), a la amistad, la diversión, el trabajo en equipo y a la condición física (Gould y cols., 1985; Weinberg y cols., 2000). Por su parte, las mujeres jóvenes australianas dan más énfasis al aprendizaje de las habilidades y a la orientación a la tarea (Longhurst y Spink, 1987).

Por otro lado, los hombres, le dan mayor importancia a la orientación al logro, al estatus (Gould y cols., 1985), a la competición (Rosich, 2005; Weinberg y cols., 2000), a la liberación de energía (Weinberg y cols., 2000) y tienen metas más orientadas al ego (Longhurst y Spink, 1987). El efecto de la competitividad y la orientación al triunfo no tuvo interacción con el género, en el estudio de Hellandsig (1998), ya que los hombres obtuvieron puntajes mayores que las mujeres en esas escalas independientemente del nivel de rendimiento en el deporte.

Los resultados de Petherick y Weigand (2002), revelaron que los hombres fueron significativamente más motivados extrínsecamente que las mujeres; específicamente, los varones obtuvieron mayor puntaje en introspección y regulación externa que las mujeres. Por su parte, Ryckman y Hamel (1995), investigaron los motivos relacionados a la participación en deportes de equipo organizados entre hombres y mujeres, encontrando que las mujeres que tienen gran adherencia en el deporte, tienen más fuertes actitudes competitivas basadas en el desarrollo personal de sus metas y menores necesidades de atención por parte de otros que las mujeres con bajos niveles de adherencia o compromiso; además que la hipercompetición fue el único predictor de adherencia para hombres.

\section{Nivel de participación}

Al dividir una muestra de universitarios en grupos de principiantes, avanzados y expertos, Pavón y cols. (2003), hallaron que los expertos centran su interés en la competición, la capacidad personal, la 
consecución del placer y las relaciones sociales; para los avanzados el principal motivo fue la aventura y para los principiantes los motivos relacionados con la forma física y la imagen personal tales como perder peso y compensar la actividad cotidiana. Además, cuanto mayor es el nivel de práctica, mayor es el interés demostrado hacia los valores competitivos y sociales del deporte, mientras que en los niveles inferiores predominan las motivaciones cercanas al cuidado de la imagen personal y a la concepción del deporte como elemento indispensable para mejorar la calidad de vida.

Tabernero (1998), indica que conforme aumenta el nivel de práctica, los deportistas valoran más las recompensas extrínsecas; mientras que otros autores (Dishman, 1985, citado por Barrios, 2001; Smith y Storant, 1997) expresan que los motivos iniciales para la participación tienden a ser más extrínsecos (control de peso, apariencia), mientras que los motivos relacionados con la adherencia tienden a ser más intrínsecos (disfrute, competencia).

\section{Diferentes tipos de actividad}

Al inicio del presente documento, quedó evidenciada la diferencia entre deporte y ejercicio, pero, ¿existen diferencias en los motivos para participar en estas actividades? Efectivamente, una buena parte de los deportistas tiene entre sus motivaciones más importantes llegar a conseguir un máximo rendimiento que les pueda proporcionar, además de la satisfacción personal, beneficios económicos y/o sociales, a la vez que valora sus resultados como el objetivo final de su etapa deportiva (Rosich, 2005), aparte que se reportaron más motivados intrínsecamente, así como para el reto y el disfrute (Kilpatrick, Hebert y Batolomew, 2003). Según Isogai y cols. (2004), los participantes en deportes individuales poseen mayor orientación a la tarea y más fuerte tendencia a dar importancia al rendimiento personal que los participantes en deportes colectivos. Las razones intrínsecas más importantes en el deporte competitivo fueron divertirse y mejorar las habilidades, mientras que los extrínsecos fueron estar en forma y el reconocimiento/estatus (Weinberg y cols., 2000).

Frederick y Ryan (1992), estudiaron los factores de motivación según la principal actividad física que realizaron adultos envueltos en dos clases generales: deporte y ejercicio o acondicionamiento, ya que ambas actividades tienen diferentes motivaciones por interés intrínseco y competición. Los resultados mostraron que los participantes en deportes individuales tienen más alto interés-disfrute y motivación a la competencia que los participantes en grupos de fitness $\boldsymbol{o}$ acondicionamiento; mientras que estos últimos expresaron mayor motivación relacionada al cuerpo, a su imagen corporal.

Kilpatrick y cols. (2003), encontraron que la motivación al ejercicio fue más extrínseca y enfocada en la apariencia, mantenimiento del peso y el estrés. Luego de un programa de trabajo aeróbico en mujeres, la satisfacción en cuanto a la imagen corporal y autopercepción no se diferenciaron entre aeróbicos de step, programa de danza aeróbica y el grupo control (Asci y cols., 1998).

\section{Cultura}

Así como se han estudiado las diferencias en el tipo de actividad física, la necesidad de incluir elementos culturales al considerar la motivación deportiva se ha indicado y ha llevado a un reciente interés en los estudios interculturales en motivación deportiva. Duda y Hayashi (1998), expresan la importancia de identificar el concepto de cultura y de variables culturales que influencian la motivación. Existe una necesidad por mayor investigación del impacto de la variación cultural en las conductas psicológicas y la predicción de sus interrelaciones (Gano-Overway y Duda, 2001). En la mayoría de estudios culturales se han realizado con dos diferentes poblaciones, o con una población que complemente el estudio previo realizado con otras. Por ejemplo, se ha expresado que las culturas del orientales tienden a evaluar el éxito en el deporte en términos generales que incorporan el rendimiento personal y la comparación con otros, mientras los americanos lo hacen con cada aspecto por aparte; los participantes estadounidenses tienen mayor orientación a la tarea que los japoneses y 
éstos, mayor orientación al ego que los americanos (Isogai y cols., 2003). Los hombres blancos norteamericanos definen el éxito por medio de la comparación social más que los adolescentes negros $\boldsymbol{e}$ hispanos, mientras que las mujeres y adolescentes hispanas se orientan a la tarea tanto en situaciones de éxito como de fracaso más que en cualquier otro grupo (Gano-Overway y Duda, 2001). Las razones para participar entre australianos, norteamericanos y jóvenes de Nueva Zelanda, fueron generalizadas y oscilaron entre estatus/emoción, desarrollo personal, afiliación, diversión y estética. Específicamente, los estadounidenses dieron mayor importancia a la diversión / condición física, trabajo de equipo y competición, así como al gasto energético que los otros dos grupos (Weinberg y cols., 2000). Los niños en China comparten muchos de los motivos para participar en deporte que los estadounidenses, entre ellos diversión, condición física, competencia, mejoramiento de sus habilidades y motivos sociales. Además los jóvenes chinos y de Hong Kong enfatizan el aprendizaje de enseñanzas para convertirse en atletas profesionales o profesionalizarse como educadores físicos (Wang y Weise-Bjornstal, 1996; Yan y McCullagh, 1997; Lindner, 1999). Finalmente, muestras de deportistas europeos (jóvenes italianos e ingleses), expresaron como motivos de participación: el disfrute, condición física/salud, social, competición, habilidades, estatus y amistad (Mason, 1995, citado por Nigg, 2003).

\section{CONCLUSIONES:}

- Los motivos de participación en la actividad física son de diversa índole: competición, capacidades personales, aventura y diversión, aspectos sociales, forma física e imagen personal y salud médica, entre otros.

- Los niveles de satisfacción personal provocados por el ejercicio son de diversa índole: física, psíquica/psicológica y social y se ven influenciados por factores personales y ambientales.

- Los niños deportistas se acercan más al deporte por motivos intrínsecos que extrínsecos como mejorar nivel, divertirse y hacer nuevos amigos.

- Al llegar la adolescencia disminuye la actividad física en ambos sexos pero principalmente en la mujeres, en esta etapa los principales motivos son: diversión, aprender nuevas habilidades, mejorar su forma física, competir y motivos sociales como cooperar con otros, obtener estatus, prestigio y reconocimiento.

- Si se desea un incremento en el nivel de motivación de estudiantes universitarios para la práctica, se debe dar prioridad a las habilidades y capacidades personales básicas, en la diversión y aventura, en las relaciones grupales y en la forma física e imagen personal; no tanto en la popularidad, la recompensa externa y la competición.

- Está claro que las enfermedades son una barrera para que el adulto mayor se ejercite, y la inactividad o sedentarismo produce deterioro en la condición física, por eso, motivos de salud física y mental son su prioridad.

- Las mujeres, suelen tener metas de control de peso y mejorar su apariencia, han demostrado ser más orientadas a lo social (la relación de pares y un fuerte énfasis social a la amistad, la diversión, el trabajo en equipo y a la condición física).

- Los hombres se enfatizan en la competición y en la obtención de estatus.

- Cuanto mayor es el nivel de práctica, mayor es el interés demostrado hacia los valores competitivos y sociales del deporte, mientras que en los niveles inferiores predominan las motivaciones al cuidado de la imagen personal y a la concepción del deporte como elemento indispensable para mejorar la calidad de vida.

- Los motivos para el ejercicio fueron más extrínsecos: enfocados en la imagen corporal, la apariencia, el mantenimiento del peso y el estrés.

- Las razones intrínsecas más importantes en el deporte fueron la competición, divertirse, mejorar las habilidades, estar en forma y el reconocimiento social / estatus.

- Los participantes norteamericanos, asiáticos y europeos prefieren indiferentemente, los motivos de diversión, competencia, condición física y aprender habilidades. 
El propósito de este documento fue orientar a los profesionales de la actividad física en las variables que determinan los motivos de participación, con el fin de maximizar la efectividad de los programas y promover el disfrute de su participación en las competiciones deportivas y así alargar su ciclo de vida deportiva competitiva. Una población indispensable en este trabajo es la niñez, ya que aunque el inicio en el deporte se da primordialmente influenciado por sus padres que les desean inculcar la práctica deportiva como actividad habitual de sus vidas, para tenerlos en algo saludable, hacerlos más disciplinados, distraerlos y en algunos casos para prevenir más adelante problemas de tipo social como drogas y alcohol, el entrenador debe ser capaz de motivar al niño para que logre mantenerse en el juego y para obtener el disfrute mientras lo realiza, para que no sea una imposición sino, para que la motivación para practicarlo sea dictada por el simple echo de practicarla, por la felicidad y la satisfacción que genera el ejercitarse.

Se ha comprobado que cuanto mayor participación activa de los niños en el deporte, hay una mayor posibilidad de continuar activos en edades avanzadas; en la sociedad actual, en específico, los niños no solo tienen menos condición física que los de pasadas generaciones, sino que también llevan estilos de vida más sedentarios e inactivos. Si este patrón de inactividad continúa hasta la edad adulta, se podrá estar en el futuro, en presencia de la generación con mayor riesgo de enfermedades crónicas y degenerativas.

Por ello, los entrenadores de equipos de iniciación deportiva, deberán incluir en sus planes de trabajo actividades que no sean tediosas ni repetitivas; tienen que hallar la manera de que sus jugadores disfruten cada una de éstas, que le encuentren el gusto y paralelamente, ir en búsqueda de objetivos de enseñanza y desarrollo así como la mejora del rendimiento de habilidades básicas y específicas. Es un área que se debe fortalecer directamente para quienes trabajan con programas deportivos y quienes son psicólogos deportivos.

\section{REFERENCIAS}

Alderman, R. B., y Wood, N. L. (1976). An analysis of incentive motivation in young Canadian athletes. Canadian Journal of Applied Sports Science, 1, 169-176.

Asci, F. H., Kin, A. S. y Kosar, N. (1998). Effect of participation in an 8 week aerobic dance and step aerobics program on physical self-perception and body image satisfaction. International Journal of Sports Psychology, 29, 366-375.

Babbkes, M.L. y Weiss, M.R. (1999). Parental influence on children's cognitive and affective responses to competitive soccer participation. Pediatric Exercise Science, 11, 44-62.

Barrios, D. (2001). Motivación hacia la práctica del ejercicio en corredores cubanos. http: www.efdeportes.com/Revista Digital, Buenos Aires (6), 31. 6 nov 2004.

Bergerone, C., Cei, A. y Ceridono, D. (1985). Motivations in the trainer-child relationship. International Journal of Sport Psychology, 16, 150-157.

Biddle, S. J. H. (1999). Motivation and Perceptions of Control: Tracing its development and plotting its future in exercise and sport psychology. Journal of Sport and Exercise Psychology, 21, 123.

Blasco, T., Capdevilla, Ll., Pintanel, M., Valiente, L. y Cruz, J. (1996). Evolución de los patrones de actividad física en estudiantes universitarios. Revista de Psicología del deporte, 9-10, 51-63. 
Brunel, P. (2003). Influence of goal orientations on situational motivation in sport: the mediating role of contextual motivation. Journal of Sport and Exercise Psychology (supplement), 25, S31.

Butt, D.S. y Cox, D.N. (1992). Motivational patterns in Davis Cup, University and recreational tennis players. International Journal of Sport Psychology, 23, 1-13.

Campbell, P., MacAuley, D., McCrum, E. y Evans, A. (2001). Age differences in the motivational factors for exercise. Journal of Sport and Exercise Psychology, 23, 191-199.

Carron, A.V. (1996). Social influence and exercise: A metaanalysis. Journal of Sport and Exercise Psychology, 18, 1-16.

Cale, L. (1996). An assessment of the physical activity levels of adolescent girls - implications for Physical Education. European Journal of Physical Education, 1, 46-55.

Cervelló, E.M. y Santos-Rosa, F.J. (2001). Motivation in sport: An achievement goal perspective in young Spanish recreational athletes. Perceptual and Motor Skills, 92, 527-534.

Chi, L., Lin, C.Y., Hung, C.H. y Liu, C.L. (2002). Relationship of goal orientation, perceived motivational climate, and perceived ability to coping strategies among elite soccer players. Journal of Sport and Exercise Psychology (supplement), 24, S44.

Chi, L., Tung, C.N., Liu, Y.C. y Feng.L.H. (2002). The relationship of perceived motivational climate, intrinsic motivation, and satisfaction in physical education classes. Journal of Sport and Exercise Psychology (supplement), 24, S45.

Cookburn, C. (2000). Las opiniones de chicas de 13 y 14 años sobre la Educación Física en las escuelas públicas británicas. Estudio realizado en los condados de Hampshire y Cambridgeshire. Apunts: Educación Física y Deportes, 62, 91-101.

Cress, M. E., Buchner, D.M., Phohaska, T., Rimmer, J., Brown, M., Macera, C., DiPietro, L. y Chodzko-Zajko, W. (2005). Best practices for physical activity programs and behavior counseling in older adult populations. Journal of Aging and Physical Activity, 13, 61-74.

Cruz, J. (1997). Factores motivacionales en el deporte infantil y asesoramiento psicológico a entrenadores y padres. Psicología del deporte. (p.147-163). Madrid, España: Editorial Síntesis, S.A.

Deci, E. L. y Ryan, R. M. (1985). Intrinsic motivation and self-determination in human behavior. New York: Plenum.

Duda, J. L. y Hayashi, C. T. (1998). Measurement issue in cross-cultural research within sport and exercise psychology. En Isogai H., Brewer, B. W., Cornelius, A. E., Etnier, J. y Tokunaga, M. (2003). International Journal of Sport Psychology, 34, 80-93.

Durand, M. (1988). El niño y el deporte. (2da Edición). Barcelona, España: Ediciones Paidos.

Etnier, J. L., Sidman, C. L. y Hancock, L. C. (2004). An examination of goal orientation profiles and motivation in adult team sport. International Journal of Sport Psychology, 35, 173-188.

Frederick, C.M. y Ryan, R.M. (1992). Differences in motivation for sports and exercise and their relations with participation and mental health. Journal of Sport Behavior, 16, 124-129. 
Gano-Overway, L. A. y Duda, J. L. (2001). Personal theories of achievement motivations among African and white mainstream American athletes. International Journal of Sport Psychology, $32,335-354$.

Gill, D.L., Gross, J.B. y Huddleston, S. (1983). Participation motivation in youth sports. International Journal of Sport Psychology, 14, 1-14.

Glenn, S., Horn, T., Campbell W. y Burton, D. (2003). Interactive effects of perceived coach and peer leadership styles on young athletes' psychosocial status and perceptions of team motivational climate. Journal of Sport and Exercise Psychology (supplement), 25, S7.

González, G., Tabernero, B. y Márquez, S. (2000). Análisis de los motivos para participar en fútbol y en tennis en la iniciación deportiva. Revista motricidad, 6, 47-66.

Gould, D. (1982). Sport psychology in the 1980s: status, direction, and challenge in youth sport research. Journal of Sport Psychology, 4, 203-218.

Gould, D., Feltz, D. y Weiss, M. (1985). Motives for participating in competitive youth swimming. International Journal of Sport Psychology, 16, 126-140.

Hagger, M., Cale, L. y Almond, L. (1997). Children`s physical activity levels and attitudes towards physical activity. European Physical Educator Review, 3(2), 144-164.

Harter, S. (1981). A model of competence motivation in the mastery motivation in children: Individual differences and developmental change. En Collins (Eds.), Minnesota Symposium on Child Psychology (Vol. 14). Hillsdale, NJ: Erlbaum.

Hellandsig, E. T. (1998). Motivational predictors of high performance and discontinuation in different types of sports among talented teenage athletes. International Journal of Sport Psychology, 29, 27-44.

Huang, M.Y., Chou, C.C., Lu, C.Y., Chen, C.H., Yeh, Y.T., Chang C.Y., Lin, C.W., Lin, M.H., Wang Y.H. y Wu, Y.C. (2003). Perception of the physical education learning environment and intrinsic motivation in physical education: a case on high school students in Northern Taiwan. Journal of Sport and Exercise Psychology (supplement), 25, S72.

Isogai, H., Brewer, B. W., Cornelius, A. E., Etnier, J. y Tokunaga, M. (2003). A cross-cultural analysis of goal orientation in American and Japonese Physical Education students. International Journal of Sport Psychology, 34, 80-93.

Kilpatrick, M., Hebert, E. y Bartholomew, J. (2003). Motivation for physical activity: differentiating motives for sport and exercise participation. Journal of Sport and Exercise Psychology, 25, supplement, S80.

Kim, M.S., Chang, D.S. y Gu, H. (2003a). Difference in skill levels and gender in achievement goals, perceptions of motivational atmosphere, and motivation in sport. Journal of Sport and Exercise Psychology (supplement), 25, S81.

Kim, B. J., Williams, L. y Gill, D. L. (2003). A cross-cultural study of achievement orientation and intrinsic motivation in young USA and Korean athletes. International Journal of Sport Psychology, 34, 168-184. 
Lindner, K.J. (1999). Sport participation and perceived academia performance of school children and youth. Pediatric Exercise Science, 11, 129-143.

Longhurst, K., y Spink, K.S. (1987). Participation motivation of Australian children involved in organized sport. Canadian Journal of applied Sport Sciences, 12, 24-30.

Maehr, M.L. y Nicholls, J.G. (1980). Culture and achievement motivation: A second look. En Warren, N. (Ed), Studies in cross-cultural psychology (Vol 2, pp. 221-267). New York: Academic Press.

Martínez, R., Andrade, A. y Salguero, A. (2005). Análisis factorial del cuestionario de motivos de participación deportiva de Gill y cols. (1983) Adaptado a una muestra de jóvenes futbolistas. Memoria del X Congreso Nacional y Andaluz de Psicología de la Actividad Física y el Deporte (291-302). Málaga, España.

McCarthy, K., White, S.A. y Amorose, A.J. (2003). Achievement goals, intrinsic motivation, and sport commitment in female volleyball players. Journal of Sport and Exercise Psychology (supplement), 25, S97.

Morilla, M. (1994). El papel del entrenador en la motivación deportiva. El entrenador Español de Fútbol, 60, 26-31.

Nicholls, J.G. (1984). Achievement motivation: Conceptions of ability, subjective experience, task choice, and performance. Psychological Review, 91, 328-346.

Nicholls, J.G. (1989). The competitive ethos and democratic education. Cambridge, MA, EE.UU.: Harward University Press.

Nigg, C. R. (2003). Do sport participation motivations add to the ability of the transtheoretical model to explain adolescent exercise behavior? International Journal of Sport Psychology, 34, 208225.

Pavón, A. I., Moreno, J. A., Gutiérrez, M. y Sicilia, Á. (2003). Interés y motivaciones de los universitarios: diferencias en función del nivel de práctica. Cuadernos de Psicología del Deporte, 3(1), 33-43.

Pelletier, L.G., Fortier, M.S., Vallerand, R.J., Tuson, K.M., Brière, N.M. y Blias, M.R. (1995). Toward a new measure of intrinsic motivation, and amotivation in sport: the Sport Motivation Scale (SMS). Journal of Sport and Exercise Psychology, 17, 35-53.

Petherick, C. M., y Weigand, D. A. (2002). The relationship of dispositional goal orientations and perceived motivational climates on indices of motivation in male and female swimmers. International Journal of Sport Psychology, 33, 218-237.

Recours, R.A., Souville, M. y Griffet, J. (2004). Expressed motives for informal and club/association-based sports participation. Journal of Leisure Research, 36, 1, 1-22.

Rosich, M. (2005). Estudio sobre la percepción de satisfacción en el deporte en el ámbito competitivo en una muestra de universitarios. Memoria del X Congreso Nacional y Andaluz de Psicología de la Actividad Física y el Deporte. (pp. 632-641). Málaga, España. 
Rudisill, M.E., Wall, S.J., Parish, L.E., St Onge, P. y Goodway J.D. (2003). Effectiveness of a preschool mastery-motivational-climate motor-skill-development intervention program: gender equity issues. Journal of Sport and Exercise Psychology (supplement), 25, S113.

Ryckman, M. R. y Hamel, J. (1995). Male and female adolescents' motives related to involvement in organized team sports. International Journal of Sport Psychology, 26, 383-397.

Seifriz, J.J., Duda, J.L. y Chi, L. (1992). The relatioship of perceived motivational climate to intrinsic motivations and beliefs about success in basketball. Journal of Sport and Exercise Psychology, $14,375-391$.

Simons, J., Dewitte, S. y Lens, W. (2003). "Don't do it for me. Do it for yourself!" Stressing the personal relevance enhances motivation in physical education. Journal of Sport and Exercise Psychology, 25, 145-160.

Skubic, E. (1956). Studies of little and middle league baseball. Research Quarterly. 26, 97-110.

Smith, A.L., Ullrich-French, S., Walter, II, E.G. y Hurley K.S. (2003). Peer relationship profiles and motivation in youth sport. Journal of Sport and Exercise Psychology (supplement), 25, S6-S7.

Smith, C. y Storandt, M. (1997). Physical activity participation in older adults: A comparison of competitors, noncompetitors and nonexersice. Journal of Aging and Physical Activity, 5, 98110.

Tabernero, B. (1998). Motivos para practicar tenis en la iniciación deportiva. Ponencia presentada en memoria del II Congreso Internacional sobre la Enseñanza de la Educación física y el Deporte Escolar, Málaga, Instituto Andaluz del deporte, España.

Taylor, W. C., Blair, S. N., Cummings, S. S., Wun, C. C. y Malina, R. M. (1999). Childhood and adolescent physical activity patterns and adult physical activity. Medicine and Science in Sports and Exercise, 31(1), 118-123.

Tercedor, P. y Delgado. M. (1998). El sedentarismo en los escolares: estudio en población de $5^{\circ}$ curso de Educación Primaria. En García, A., Ruiz, F y Casimiro, A. J. (Eds.), Memoria del II Congreso Internacional sobre la Enseñanza de la Educación física y el Deporte Escolar, (pp. 277-280). Málaga, Instituto Andaluz del deporte, España.

Trail, G. T., Anderson, D. F. y Flink, J. (2002). Examination of gender differences in importance of and satisfaction with venue factors at intercollegiate basketball games. International Sports Journal, 6(1), 51 - 64 .

Treasure, D. C. y Roberts, G. C. (1998). Relationship between female adolescent's achievement goal orientations, perceptions of the motivational climate, belief about success and sources of satisfaction in basketball. International Journal of Sport Psychology, 29, 211-230.

Trew, K., Scully, D., Kremer, J. Y Olge, S. (1999). Sport, leisure and perceived self-competence among male and female adolescents. European Physical Education Review, 5(1), 53-73.

Valdés, H. M. (1996). La preparación psicológica del deportista. Mente y rendimiento humano. (pp.54-57). España: INDE publicaciones. 
Vlachopoulos, S., Biddle, S. y Fox, K. (1996). A social-cognitive investigation into the mechanisms of affect generation in children's physical activity. Journal of Sport and Exercise Psychology, $18,174-193$.

Wang, J. y Weise-Bjornstal, D. M. (1996). The relationship of school type and gender to motives for sport participation among youth in the People's Republic of China. International Journal of Sport Psychology, 28, 13-24.

Weigand, D.A. y Broadhurst, C.J. (1998). The relationship among perceived competence, intrinsic motivation, and control perceptions in youth soccer. International Journal of Sport Psychology, 29, 324-338.

Weinberg, R.S. y Gould, D. (1996). Fundamentos de psicología del deporte y el ejercicio físico. (pp. 230-250). Barcelona, España: Editorial Ariel, S.A.

Weinberg, R., Tenenbaum, G., McKenzie, A., Jackson, S., Anshel, M., Grove, R. y Fogarty, G. (2000). Motivation for youth participation in sport and physical activity: relationships to culture, self-reported activity levels, and gender. International Journal of Sport Psychology, 31, 321-346.

Weiss, M.R. y Smith, A.L. (2002). Friendship quality in youth sport: Relationship to age, gender, and motivational variables. Journal of Sport and Exercise Psychology, 24, 420-437.

White, S.A. (1998). Adolescent goal profiles, perceptions of the parental-initiated motivational climate, and competitive trait anxiety. The Sport Psychologist, 12, 16-28.

Williams, L. y Cox, A. (2003). The relationship among social motivational orientations, perceived social belonging, and motivation-related outcomes. Journal of Sport and Exercise Psychology (supplement), 25, S7-S8.

Yan, J. H. y McCullagh, P. (1997). Cultural Influence on Youth's Motivation of Participation in Physical Activity. Journal of Sports Behavior, 27, 4, 378-390.

Yilmaz, H. y Akandere, M. (2003). Effects of sports activities on life levels for women. International Journal of Sport Psychology, 34, 322-328.

Fecha de recepción del artículo: abril del 2006.

Fecha de aceptación del artículo: abril del 2006.

Fecha de publicación del artículo: 20 de julio del 2006. 